\title{
Prevalencia del consumo de sustancias psicoactivas y la aparición de la enfermedad mental en adolescentes ${ }^{1}$
}

Carlos Marín Madrigal ${ }^{2}$ José Calderón Barboza ${ }^{3}$

\section{Institución: Hospital Nacional Psiquiátrico}

Pbro. Manuel Antonio Chapuí y Torres

\begin{abstract}
RESUMEN
El objetivo de este trabajo es presentar los resultados de una investigación secundaria acerca de la prevalencia del consumo de sustancias psicoactivas (SPA) en la población adolescente con enfermedad mental en comparación con los adolescentes que no padecen enfermedad mental. Se formuló una pregunta según el acrónimo "PICO" (pacientes, intervención, comparación, resultados); posteriormente, se buscó información en distintas bases de datos sugeridas en el curso de Práctica Clínica Basada en la Evidencia, impartido por el Programa de Colaboración para la Investigación de Enfermería Basada en la Evidencia de Costa Rica (CIEBE-CR). Para la búsqueda de información se efectuó una revisión de artículos científicos en Medline Plus, PudMed y EBSCO Host, con el fin de recuperar la mayor cantidad de información. Después de establecer los criterios de inclusión y exclusión y realizar un análisis crítico, se seleccionó cuatro documentos que dieron respuesta a la pregunta planteada. Se detectó 58 documentos relacionados con el tema de los cuales solo cuatro cumplían criterios de calidad que establece el Critical Appraisal Skills Programme español (CASPe) para responder a la pregunta clínica. Se evidencia cómo en la niñez la psicopatología se reconoce cada vez más como un factor de riesgo en cuanto al uso de sustancias psicoactivas. Se concluye que los adolescentes con patología mental tienen una mayor prevalencia al uso de sustancias psicoactivas en comparación con los adolescentes que no padecen enfermedad mental.
\end{abstract}

Palabras clave: adolescente; prevalencia; sustancias psicoactivas.

\footnotetext{
${ }^{1}$ Fechas de recepción: 12 de agosto 2017

Fecha de aceptación: 4 de diciembre 2017

${ }^{2}$ Enfermero. Hospital Nacional Psiquiátrico Pbro. Manuel Antonio Chapuí y Torres. Costa Rica. Correo electrónico: cemm2765@gmail.com

${ }^{3}$ Auxiliar de enfermería. Hospital Nacional Psiquiátrico Pbro. Manuel Antonio Chapuí y Torres. Costa Rica. Correo electrónico: josecalde66@yahoo.com
} 


\title{
Prevalence of psychoactive substance use and mental illness in adolescents ${ }^{1}$
}

\author{
Carlos Marín Madriga ${ }^{2}$ \\ José Calderón Barboza ${ }^{3}$
}

Institution: Hospital Nacional Psiquiátrico

Pbro. Manuel Antonio Chapuí y Torres

\begin{abstract}
The objective of this paper is to present the results of secondary research on the prevalence of psychoactive substance use (PSA) in the adolescent population with mental illness compared to adolescents who do not suffer from mental illness. A question was formulated according to the acronym "PICO" (patients, intervention, comparison, results); Subsequently, information was sought in different databases suggested in the Clinical Practice Based on Evidence course, taught by the Collaboration Program for Nursing Research Based on the Evidence of Costa Rica (CIEBE-CR). For the search of information, a review of scientific articles was carried out in Medline Plus, PudMed and EBSCO Host, in order to recover the greatest amount of information. After establishing the inclusion and exclusion criteria and performing a critical analysis, four documents that answered the question posed were selected. It was detected 58 documents related to the topic of which only four met quality criteria established by the Spanish Critical Appraisal Skills Program (CASPe) to answer the clinical question. It is evidenced how, in childhood, psychopathology is increasingly recognized as a risk factor in the use of psychoactive substances. It is concluded that adolescents with mental pathology have a higher prevalence of the use of psychoactive substances in comparison with adolescents who do not suffer from mental illness.
\end{abstract}

Keywords: adolescent; prevalence; psychoactive-substances.

\footnotetext{
${ }^{1}$ Date of receipt: August 12, 2017

Date of acceptance: December 4, 2017

${ }^{2}$ Nurse. Hospital Nacional Psychiatric Hospital Pbro. Manuel Antonio Chapuí y Torres. Costa Rica. E-mail: cemm2765@gmail.com

${ }^{3}$ Nursing assistant. Hospital Nacional Psiquiátrico Pbro. Manuel Antonio Chapuí y Torres. Costa Rica. E-mail: josecalde66@yahoo.com
} 


\title{
Revista Electrónica Enfermeria Actual en costa Rica
}

\section{Prevalência de uso de substâncias psicoativas e doenças mentais em adolescentes ${ }^{1}$}

\author{
Carlos Marín Madrigal ${ }^{2}$
}

José Calderón Barboza ${ }^{3}$

\section{Instituição: Hospital Nacional Psiquiátrico}

Pbro. Manuel Antonio Chapuí y Torres

\section{RESUMO}

O objetivo deste trabalho é apresentar os resultados da pesquisa secundária sobre a prevalência do uso de substâncias psicoativas (PSA) na população adolescente com doença mental em comparação com adolescentes que não sofrem de doença mental. Uma pergunta foi formulada de acordo com a sigla "PICO" (pacientes, intervenção, comparação, resultados); Posteriormente, a informação foi buscada em diferentes bancos de dados sugeridos no curso de Prática Clínica Baseada na Evidência, ministrado pelo Programa de Colaboração para Pesquisa de Enfermagem com base na Evidência da Costa Rica (CIEBE-CR). Para a busca de informações, foi realizada uma revisão de artigos científicos no Medline Plus, PudMed e EBSCO Host, a fim de recuperar a maior quantidade de informações. Depois de estabelecer os critérios de inclusão e exclusão e realizar uma análise crítica, foram selecionados quatro documentos que responderam à pergunta colocada. Foram detectados 58 documentos relacionados ao tema, dos quais apenas quatro critérios de qualidade estabelecidos pelo Programa Espanhol de Competências de Avaliação Crítica (CASPe) para responder à questão clínica. É evidenciado como, na infância, a psicopatologia é cada vez mais reconhecida como fator de risco no uso de substâncias psicoativas. Conclui-se que os adolescentes com patologia mental têm maior prevalência do uso de substâncias psicoativas em comparação com adolescentes que não sofrem de doença mental.

Palavras-chave: adolescente; prevalência; substâncias-psicoativas.

\footnotetext{
${ }^{1}$ Data de recepção: 12 de agosto de 2017

Data de aceitação: 4 de dezembro de 2017

${ }^{2}$ Enfermeira. Hospital Nacional Psiquiátrico Pbro. Manuel Antonio Chapuí y Torres. Costa Rica. Correio eletrônico: cemm2765@gmail.com

${ }^{3}$ Auxiliar de enfermagem. Hospital Nacional Psiquiátrico Pbro. Manuel Antonio Chapuí y Torres. Costa Rica. Correio eletrônico: josecalde66@yahoo.com
} 


\section{Revista Electrónica Enfermeria Actual en costa Rica}

\section{INTRODUCCIÓN}

En los últimos años la atención de la salud mental ha enfrentado cambios considerables, debido a las características poblacionales que se reflejan en cambios en el comportamiento epidemiológico del sistema de salud; por lo tanto, la atención en psiquiatría en Costa Rica también se ha visto afectada con el paso del tiempo, con la implementación de nuevas estrategias de atención, lo cual amerita modificar la intervención tanto a nivel hospitalario, como comunitario.

En cuanto a la patología dual, es definida como la coexistencia de dos trastornos que pueden ser o no independientes, pero sí necesariamente interactivos, respecto de la presentación concomitante de un trastorno por uso de sustancias y otro trastorno mental ${ }^{1}$. En los últimos diez años el término ha ido incursionando en el ámbito de la comunidad de profesionales de la salud mental y de la asistencia a los problemas derivados de la drogodependencia; sin embargo, es un diagnóstico discriminado y hasta rechazado como tal ${ }^{2}$.

A pesar de lo anterior, dicho panorama parece estar cambiando: por ejemplo, la Sociedad Española de Patología Dual ha realizado diferentes jornadas para retomar el tema, entre las que se identifica el Estudio de Prevalencia de la Patología Dual en la Comunidad de Madrid, en el marco de la XXXVI Jornadas Nacionales de Socidrogalcohol, realizadas en Salamanca en el 2009 en centros de atención a drogodependientes y centros de salud mental: al respecto, de la población estudiada (433 individuos sobre 837), el 51,7\% cumplía criterios para patología dual actual, ya que existía la presencia de abuso/dependencia de alcohol u otras drogas más diagnóstico establecido de trastorno mental y/o trastorno de la personalidad; en los centros de atención a drogodependientes, el 61,7\% (387 de 629 personas) cumplen criterios de patología dual actual; es decir, dos de cada tres personas con adicción a drogas presentan algún trastorno mental y/o de la personalidad ${ }^{3}$, situación sobre la que se plantea el objetivo de esta investigación.

Actualmente, el trastorno por dependencia no es un problema individual, sino social en el que el consumismo, el estrés, la soledad, el aislamiento, la depresión, la violencia, la competitividad provoca que las personas busquen un "alivio" a esos malestares con sustancias ilícitas y fomentando la dependencia ${ }^{4}$. Por lo anterior, es que la Organización Mundial de la Salud (OMS) ${ }^{5}$ y la Asociación Psiquiátrica Americana, establece esta enfermedad como crónica, debido a los cambios permanentes que produce a nivel cerebral por la neuroadaptación, lo que permite una vulnerabilidad orgánica con síntomas de riesgo tanto individual como colectivo, por las alteraciones conductuales que esto genera, así como delitos, accidentes de tránsito entre otros.

En el caso de esta drogodependencia, se asocia a diferentes trastornos mentales y de la personalidad, por lo que el pronóstico es muy conservador, por la falta de apego al tratamiento, recaídas, secuelas de tipo orgánico y esto implica un gasto público elevado 6 .

Así pues, la concurrencia de patología adictiva y psiquiátrica en adolescentes constituye uno de los desafíos más interesantes para los próximos años, por lo que para el profesional es importante profundizar y mejorar los conocimientos de la enfermedad mental en esta área para poder establecer estrategias terapéuticas que propicien un manejo adecuado de esta enfermedad.

En relación con lo anterior, actualmente, en el Hospital Nacional Psiquiátrico el aumento de ingresos de adolescentes que sufren trastorno mental y además son farmacodependientes de sustancias psicoactivas ha 


\section{Revista Electrónica Enfermeria Actual en costa Rica}

impactado los modelos de atención, ya que los servicios de observación, el servicio de adolescentes y el programa de Nuevos Horizontes se han saturado, circunstancia que limita el ingreso de usuarios con otras patologías psiquiátricas que también ameritan atención en los servicios mencionados.

La situación mencionada lleva a los investigadores a generar una pregunta de primera línea según el acrónimo PICO ( $\mathrm{P}=$ persona o problema de interés; $\mathrm{I}=$ Intervención que se está considerando; $\mathrm{C}=\mathrm{Comparación} \mathrm{de}$ intervenciones; $\mathrm{O}=$ Variable o variables que valoren el resultado clínico).

El objetivo que se planteó fue conocer si los adolescentes con enfermedad mental son más propensos al consumo de sustancias psicoactivas en comparación con los adolescentes que no padecen enfermedad mental.

\section{MATERIALES Y MÉTODO}

La presente investigación se realiza empleando la metodología recomendada para llevar a cabo la práctica de enfermería basada en la evidencia (EBE), ya que representa "un enfoque de toma decisiones en el cual los clínicos usan la mejor evidencia disponible al atender a los pacientes para decidir cuál es la opción más óptima que tenga el paciente según Urra y otros",

1. Formulación de una pregunta en formato PICO que permita convertir esa información recopilada y que responda a esas necesidades, para así establecer las estrategias de búsqueda.

2. Búsqueda de información científica disponible

3. Análisis crítico de los datos

4. Transferencia de la evidencia a la práctica clínica

5. Evaluación

De acuerdo con el acrónimo PICO para elaborar la pregunta clínica, se detalla a continuación:

Persona o problema de interés $(\mathrm{P})$ : adolescentes.

Intervención (I): el consumo de sustancias psicoactivas.

Resultados esperados (O): favorece la aparición de enfermedades mentales.

La pregunta clínica se plantea de la siguiente forma: ¿En los adolescentes, la prevalencia del consumo de sustancias psicoactivas favorece la aparición de enfermedades mentales?

La pregunta se clasifica como de prevalencia.

Para llevar a cabo la segunda etapa referida a la búsqueda de información se efectúo una revisión de artículos científicos en Medline Plus, PudMed y EBSCO Host, con el fin de recuperar la mayor cantidad de información. 


\section{Revista Electrónica Enfermeria Actual en costa Rica}

Como descriptores, se empleó los siguientes, tanto en español como en inglés: adolescentes y enfermedad mental, sustancias psicoactivas y enfermedad mental. Dual pathology and teen, psychoactive substance consumption and mental illness.

Para esta investigación se toma como criterios de inclusión los adolescentes que oscilen entre los 12 y 18 años de edad, con diagnóstico psiquiátrico, consumidores de alguna sustancia psicoactiva, asimismo para la búsqueda se estableció los siguientes criterios de selección de documentos escritos en el idioma inglés y español, publicados en los últimos 5 años, relacionado con la temática del tema, las cuales fueron desarrolladas por los dos investigadores por separado y luego en reuniones establecidas se eligió los documentos que debían ser sometidos a un análisis crítico.

De esos documentos fueron recuperados 58 que representan los criterios de inclusión en cuanto a la revisión por título y resumen de los que, a su vez, se excluyó 43, por tanto, solo quedaron 15 publicaciones vinculadas con el uso de sustancias psicoactivas (SPA) a las cuales se les realizó el análisis crítico. Finalmente, se obtuvo cuatro publicaciones que correlacionaban el uso de SPA con la enfermedad mental en los adolescentes (figura 1).

El análisis crítico se realizó aplicando a cada documento la plantilla Critical Appraisal Skills Programme Spanish (CASPe), para luego responder a la pregunta PICO planteada.

Figura 1. Diagrama de flujo de revisión de literatura
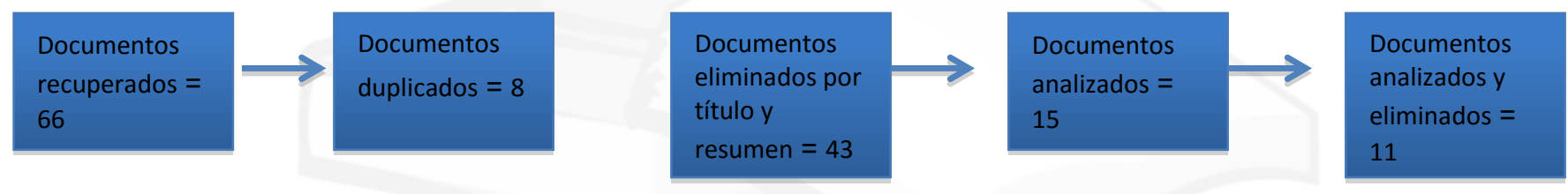

\section{Consideraciones éticas}

Durante el proceso de investigación se respetó los derechos de autor de cada uno de los artículos revisados y utilizados lo cual queda evidenciado con la citación correcta de cada uno de ellos.

\section{RESULTADO}




\section{Revista Electrónica Entermeria actualen costa Rica}

A continuación se presenta la tabla de documentos que se incluyeron en esta revisión para su análisis (tabla 1).

\section{Tabla 1. Estudios incluidos en la lectura crítica}

\begin{tabular}{|c|c|c|c|c|c|}
\hline Título & $\begin{array}{c}\text { Referencia } \\
\text { (COMPLETA) }\end{array}$ & $\begin{array}{l}\text { Tipo estudio } \\
\text { de }\end{array}$ & Intervención & Resultado & $\begin{array}{c}\text { Calidad } \\
\text { de } \\
\text { de la } \\
\text { evidencia }\end{array}$ \\
\hline $\begin{array}{l}\text { 1. Use of licit and illicit } \\
\text { substances among } \\
\text { adolescents in Brazil - A } \\
\text { national survey }\end{array}$ & $\begin{array}{l}\text { Madruga C. y } \\
\text { otros }(2012)\end{array}$ & $\begin{array}{l}\text { Cohorte } \\
\text { transversal }\end{array}$ & Encuesta & $\begin{array}{l}\text { La alta prevalencia de consumo de alcohol, } \\
\text { tabaco y otras sustancias ilícitas en los } \\
\text { adolescentes brasileños es fuerte. Los hombres } \\
\text { jóvenes con trastorno del ánimo en las áreas } \\
\text { urbanas tienen mayor riesgo de dependencia, } \\
\text { asociado a disfunción familiar. }\end{array}$ & $\begin{array}{l}2 b \\
\text { Según } \\
\text { Oxford }\end{array}$ \\
\hline $\begin{array}{l}\text { 2.Prevalencia del consumo } \\
\text { compulsivo de alcohol y } \\
\text { sustancias ilícitas en } \\
\text { pacientes hospitalizados por } \\
\text { patologías psiquiátricas }\end{array}$ & $\begin{array}{l}\text { F. Barrios, A. } \\
\text { Hurtado (2012 }\end{array}$ & $\begin{array}{l}\text { Estudio } \\
\text { descriptivo, } \\
\text { transversal, } \\
\text { retrospectivo }\end{array}$ & $\begin{array}{l}\text { Recolección de } \\
\text { datos de } \\
\text { historias clínicas }\end{array}$ & $\begin{array}{l}\text { La prevalencia encontrada de consumo de } \\
\text { sustancias ilícitas y consumo compulsivo de } \\
\text { alcohol en enfermos mentales es una situación } \\
\text { que debe ser adecuadamente abordada, para } \\
\text { ofrecer una correcta interven-ción sanitaria. }\end{array}$ & $\begin{array}{l}\text { 2b } \\
\text { Según } \\
\text { Oxford }\end{array}$ \\
\hline $\begin{array}{l}\text { 3.Clinical correlates of } \\
\text { cannabis use among } \\
\text { adolescent psychiatric } \\
\text { inpatients }\end{array}$ & $\begin{array}{l}\text { S. Lev y otros. } \\
\text { (2011) }\end{array}$ & cohorte & $\begin{array}{l}\text { Formulario con } \\
\text { datos clínicos y } \\
\text { demográficos }\end{array}$ & $\begin{array}{l}\text { Tienen una alta prevalencia, el uso de } \\
\text { sustancias puede ser un factor de riesgo para } \\
\text { trastornos psiquiátricos y podría afectar } \\
\text { significativamente el tratamiento y evolución } \\
\text { de las enfermedades psiquiátricas. El abordaje } \\
\text { con antipsicó-ticos es más prolonga-do en los } \\
\text { adolescentes con uso de cannabis. }\end{array}$ & $\begin{array}{l}2 \mathrm{~b} \\
\text { Según } \\
\text { Oxford }\end{array}$ \\
\hline $\begin{array}{l}\text { 4.Síntomas psiquiátricos } \\
\text { asociados al consumo de } \\
\text { pasta base de cocaína en } \\
\text { niños y adolescentes: un } \\
\text { estudio exploratorio en } \\
\text { Montevideo, Uruguay }\end{array}$ & $\begin{array}{l}\text { Galera } \\
(2013)\end{array}$ & $\begin{array}{l}\text { Casos } \\
\text { controles }\end{array}$ & $\begin{array}{l}\text { Autocuestio- } \\
\text { narios }\end{array}$ & $\begin{array}{l}\text { El consumo de PBC se asocia a niveles } \\
\text { mayores de problemas psiquiátricos en los } \\
\text { jóvenes. Actuar precozmente para prevenir el } \\
\text { consumo de PBC y tratar los problemas } \\
\text { externalizados en los consumidores parece } \\
\text { esencial para mejorar la salud de los jóvenes en } \\
\text { Uruguay. }\end{array}$ & $\begin{array}{l}\text { 3b } \\
\text { Según } \\
\text { Oxford }\end{array}$ \\
\hline
\end{tabular}

Fuente: elaboración propia.

De los artículos encontrados en esta revisión (véase tabla 1) se incluyó cuatro que se describen a continuación.

En cuanto al tema, en un estudio de tipo transversal se incluye una investigación sobre resultados de la primera Encuesta Nacional de Alcohol de Brasil, la cual reunió información sobre el uso de sustancias psicoactivas en 716 pacientes con edades de los 12 a 19 años de edad. Se describe una prevalencia de un 29,3\% de adolescentes que presentan depresión y un 9,7\% con déficit de atención e hiperactividad: de los primeros, el 2,02\% se asoció al uso de alcohol y el 1,78\% al uso de drogas ilegales, mientras que el déficit de atención e hiperactividad se asoció el uso de alcohol en un $0,81 \%$ y el uso de drogas ilegales en un $2,76 \%{ }^{8}$.

Asimismo en la Universidad de Bogotá en Colombia, se realizó un estudio en el que se recolectó información de historias clínicas de pacientes hospitalizados en los años 2008 y 2009 en tres instituciones psiquiátricas de los 


\section{Revista Electrónica Enfermeria Actual en costa Rica}

municipios de Cartagena y Turbaco en Colombia, para calcular la prevalencia porcentual de consumo compulsivo de alcohol, de sustancias ilícitas y de ambas con respecto a la totalidad de pacientes hospitalizados? .

Los resultados muestran que 2526 pacientes fueron ingresados en los dos años en las tres instituciones, 1086 pacientes en el 2008 y 1440 en el 2009. La prevalencia de consumo compulsivo de alcohol y sustancias ilícitas fue de $19,5 \%$, la prevalencia de consumo compulsivo de alcohol fue de 2,3\% y de consumo de sustancias ilícitas fue de $18,6 \%$. No se observó diferencia entre los dos años en ninguna de las tres evaluaciones. La sustancia ilícita de mayor consumo fue la marihuana.

El estudio señala que aproximadamente el 55\% de los adultos con patología por uso de sustancias presentan un diagnóstico psiquiátrico antes de los quince años ${ }^{9}$. Además el estudio permite identificar cómo en la adolescencia se ha detectado comorbilidad entre el trastorno disocial y el consumo de sustancias. Sin embargo la relación causal entre las patologías por uso de sustancia (TUS) y el trastorno disocial (TD) no está resuelta: por un lado, el consumo de sustancias podría aparecer a causa del trastorno disocial ${ }^{9}$, lo que complicaría su evolución. Al respecto, hay estudios que indican que la aparición de TD durante la niñez es un factor de riesgo para el posterior consumo de cannabis, además de que se postula la existencia previa de patología de conducta como factor de vulnerabilidad para presentar en la adolescencia patología por uso de sustancias.

Por otro lado, en una investigación en la que participaron 236 adolescentes ingresados en una unidad de hospitalización de adolescentes de un centro de salud mental afiliado a la universidad en Israel, durante un período de 3 años, se determinó las características clínicas en adolescentes con uso de cannabis comparados en comparación con quienes no la consumen y que son pacientes psiquiátricos en Israel: el diagnóstico más común en el grupo de consumidores de cannabis fue déficit de atención y trastornos de comportamiento disruptivo $(39,1 \%)$, y en el grupo de no consumidores de drogas, trastorno psicótico $(35,9 \%)^{10}$. El estudio concluye que la psicopatología se reconoce cada vez más como un factor de riesgo para el uso de sustancias psicoactivas: la fuerte evidencia sugiere que el uso de sustancias puede ser un factor de riesgo para trastornos psiquiátricos y podría afectar significativamente el tratamiento y evolución de las enfermedades psiquiátricas ${ }^{10}$, sin olvidar que la adolescencia es considerada una etapa de vulnerabilidad a los efectos adversos del consumo de sustancias psicoactivas en el funcionamiento neuropsicológico.

Por otro lado se dice que el cannabis es la sustancia ilícita más consumida en todo el mundo, tanto para adultos y adolescentes, cuyo uso está asociado con trastornos psiquiátricos, como la depresión, tendencias suicidas comportamiento, y la psicosis, a la que los usuarios pueden ser adolescentes especialmente vulnerables.

De igual manera, en Uruguay se realizó un estudio exploratorio de casos y controles en consumidores de pasta base de cocaína (PBC), con un nivel de significatividad estadística de 0,05 con la participación de adolescentes en Uruguay ${ }^{11}$ : hubo $\mathrm{n}=20$ casos con igual número de controles con variables de emparejamiento similares en niños menores de 15 años.

Como resultado, se observó niveles elevados de problemas asociados a comportamientos internalizados y externalizados, en comparación con el grupo testigo no consumidor. Como en los comportamientos oposicionistas $(18,0$ vs 1,6$)$ y agresivos $(18,7$ vs 6,8$)$, así como dificultades atencionales $(8,7$ vs 3,9$)$ y altos niveles de 


\section{Revista Electrónica Enfermeria Actual en costa Rica}

problemas antisociales ${ }^{11}$, lo cual permitió identificar la asociación que hay entre niveles elevados de problemas externalizados, internalizados y de atención llevan al consumo de PBC.

\section{DISCUSIÓN}

Los diferentes estudios refieren que la marihuana es una de las SPA más utilizada por los adolescentes ${ }^{12}$, la cual tiene una gran relación con la esquizofrenia ${ }^{13}$. Los adolescentes relacionan el uso con efectos como el aumento de sensaciones placenteras, mayor facilidad para la expresión de opiniones y sentimientos, aceptación por parte de sus iguales, aumento del rendimiento académico, laboral y deportivo, así como de la creatividad ${ }^{14}$. Ante la falta de información real y certera que lleve a los adolescentes a reflexionar sobre las consecuencias del uso de SPA a corto o largo plazo, caen en preconceptos y valoraciones positivas de dichas sustancias, lo cual puede ser determinante en la baja percepción de riesgo ante el consumo de SPA.

En torno al tema, es importante rescatar que la actitud más favorable de los adolescentes al consumo de drogas será más elevada cuanto mayores sean los contactos con ambientes y compañías incitadores al consumo y los contactos con drogas mantenidos por los amigos, así como el consumo propio de drogas, y las manifestaciones de búsqueda de atención y conducta desviada (transgresiones de normas, absentismo escolar, conductas disruptivas, etc. $)^{15}$.

Los adolescentes que coexisten con patología dual necesitan de una atención integrada por un solo equipo terapéutico para desarrollar terapias específicas dirigidas al paciente dual, ya que tienen una presencia epidemiológica importante, según los estudios analizados del 16\% al 60\% de los sujetos en estudio.

Estas cifras de prevalencia de comorbilidad son ampliamente variables entre los diferentes estudios, independientemente las poblaciones investigadas, esto se puede deber a diferentes factores, para nosotros uno de los factores que lleva a este amplio rango de prevalencia es la falta de consulta médica de este tipo de población, como también lo menciona Gómez y otros ${ }^{16}$; los individuos no consultan, o son consumidores inconsistentes, que permanecen sin ser reconocidos, por no presentar consecuencias médicas, laborales o legales, motivo por el que Arias y otros ${ }^{17}$ mencionan que obtener una muestra de tamaño adecuado es la mayor dificultad, dada la escasa demanda de tratamiento de los adictos.

El reconocimiento de los diferentes factores que se ven asociados a una desfavorable evolución terapéutica es de gran importancia para la identificación precoz de los pacientes que tienen más riesgo de abandonar el tratamiento para así poder bridar una mayor atención con el fin de crear un mejor apego al tratamiento, para intentar minimizar el riesgo de abandono del mismo. En este se comentan como factores de riesgo; La edad más tardía al inicio del tratamiento, el policonsumo, el diagnóstico de trastornos psicóticos y de trastorno límite de personalidad $^{18}$.

Los trastornos mentales que se inician en esta etapa de la adolescencia se deben detectar y atender oportunamente, debido a que representan uno de los factores que puede llevar a generar una conducta adictiva, por ello, la atención oportuna puede ser probablemente una estrategia efectiva de prevención contra el desarrollo de esa codependencia a las sustancias psicoactivas. Por lo anterior, tanto la prevención como la promoción son fundamentales, así como investigaciones relacionadas con el consumo de drogas y la importancia desde la 


\section{Revista Electrónica Enfermeria Actual en costa Rica}

atención primaria realizada por Casado $^{19}$, quien -por medio de revisiones de guías clínicas- persigue incluir intervenciones sobre conductas adictivas de riesgo susceptibles en adolescentes, acerca de los que es necesario brindar un abordaje en todo el sistema sanitario en coordinación con recursos comunitarios desde la atención primaria para captar situaciones y conductas de riesgo, incluyendo a la familia, procesos de desintoxicación y atención paliativa, apoyo y a los profesionales de enfermería, esenciales como agentes de salud con el compromiso social para establecer estrategias claves desde la atención primaria ${ }^{20}$.

Lo mencionado se constituye en un reto para la salud pública, desde un abordaje farmacológico y psicológico, tanto a nivel estatal como organizaciones no gubernamentales (ONG), que garanticen el trabajo de la adicción como la patología psiquiátrica de forma conjunta y simultánea.

Al ser la patología dual un tema que afecta los factores epidemiológicos a nivel nacional que van en aumento, debe tomarse en cuenta para satisfacer los procesos de rehabilitación a futuro, máxime con la dificultad que los pacientes enfrentan al no encontrar programas de tratamiento que integren la atención psiquiátrica y de farmacodependencia, lo cual se traduce en tratamientos paralelos o secuenciados que disminuyen su efectividad e incrementan sus costos. Dado lo anterior, es importante hacer hincapié en la necesidades de profundizar en la caracterización nosocomial adecuada ante la coexistencia de síntomas psicopatológicos, consumo y desintoxicación de sustancias psicoactivas con instrumentos clínicos y desarrollar estrategias terapéuticas en enfermería por medio de una formación en patología dual para los profesionales involucrados en estos procesos para así satisfacer las necesidades sanitarias.

En relación con lo mencionado, la patología dual en adolescentes representa uno de esos cambios en la atención psiquiátrica, ya que el uso de sustancias psicoactivas es un factor de riesgo para la aparición de trastornos psiquiátricos, sobre todo en la adolescencia, una etapa de vulnerabilidad ante los efectos adversos del consumo de esas sustancias en cuanto al funcionamiento a nivel, social y neuropsicológico. Tal panorama invita a fomentar programas de promoción de la salud mental dirigida a la población adolescente en educación primaria, como el de la comunidad española, el cual se constituye en un entrenamiento para mejorar un estilo de vida positivo para los adolescentes, que incluye temas como las habilidades sociales, resolución de conflictos, asertividad, autoestima y el control de impulsos.

Aunque la cultura española es muy diferente a la costarricense, para Urrutia ${ }^{21}$, quien cita a la Organización Mundial de la Salud (OMS) ${ }^{22}$, al referir que hay aspectos generales como los determinantes de la salud mental y de los trastornos mentales que incluyen no tanto las características individuales (pensamientos, emociones, comportamientos, interacciones), sino componentes sociales, culturales, económicos, políticos y ambientales y la exposición de los adolescentes a las adversidades, se convierten en un factor de riesgo que se puede prevenir; evitando los trastornos mentales a tempranas edades ${ }^{20}$. Para lograrlo, es importante involucrar a las instituciones educativas - uno de los lugares donde los adolescentes pasan gran parte de su tiempo relacionándose con sus iguales- en la etapa de prevención, aun cuando suponga el gran reto que tiene el sistema de la seguridad social para abordar los procesos educativos, el cual podría convertirse en un pilar esencial para la detección temprana de conductas de alto riesgo que se asocian al consumo de PSA, a partir de las interacciones sociales ${ }^{15}$.

Por último, dado que las patologías adictivas y psiquiátricas representan uno de los desafíos más interesantes en el desarrollo de estrategias terapéuticas adecuadas en recursos asistenciales adaptados y por profesionales 


\section{Revista Electrónica Enfermeria Actual en costa Rica}

entrenados convenientemente ${ }^{22}$, es importante para los profesionales en enfermería adquirir los conocimientos necesarios para establecer estrategias y brindar una atención integral desde la prevención hasta la desintoxicación.

\section{CONCLUSIÓN}

Tomando como fundamento la pregunta PICO, se concluye que los adolescentes con patología mental tienen una mayor prevalencia al uso de sustancias psicoactivas, hecho que se justifica por ser más vulnerables a la presión de pares, además de que buscan la aceptación por parte del grupo de iguales, por tanto, el querer vivir experiencias nuevas los puede llevar al consumo de SPA.

En los estudios revisados se observó cómo ciertas patologías mentales se asocian al uso/ abuso de sustancias psicoactivas (SPA), tanto previo como posterior a ser diagnosticados con una patología mental. La prevalencia del uso de SPA en adolescentes con patología mental según los estudios anteriormente revisados oscilan entre el 16\% al $60 \%$.

\section{Conflicto de intereses}

Los autores declaran no tener conflicto de intereses de orden personal, comercial, académico, político o financiero.

\section{Referencias bibliográficas}

1. Casas, M., et al. Trastorno bipolar asociado al uso de sustancias adictivas (patología dual). Revisión sistemática de la evidencia científica y consenso entre profesionales expertos. Actas españolas de psiquiatría. 2008; 36(1). Disponible en: https://psiquiatria.com/patologia_dual/trastorno-bipolar-asociadoal-uso-de-sustancias-adictivas-patologia-dual-revision-sistematica-de-la-evidencia-cientifica-y-consensoentre-profesionales-expertos/

2. Smith, K., Larson, M. (2003). Quality of life assessments by adult substance abusers receiving publicly funded treatment in Massachusetts. American Journal Alcohol Abuse, 29, 323-335. Disponible en: https://www.ncbi.nlm.nih.gov/pubmed/12765209

3. ACAPI. Evolución de la intervención en patología dual en la comunidad terapéutica. Normas para la presentación y publicación de trabajos en el año 1989, 46(10): 161-172

4. Bejarano J, Ugalde F, Fonseca S. El consumo de drogas en hombres y mujeres costarricenses: Análisis de una década en personas de 25 a 59 años de la población general. Revista Costarricense de Salud Pública. 2006; 15(28):29-43.

5. Organización Mundial de la Salud. Trastornos Mentales y del Comportamiento debido al consumo de Sustancias Psicotrópicas. Decima Revisión de la Clasificación Internacional de Enfermedades. Trastornos Mentales y del Comportamiento. Madrid 1992 


\section{Revista Electrónica Enfermeria Actual en costa Rica}

6. Diaz-Salabert J. Marginación Social y Drogodependencias: Intervenciones y Terapias Breves con Menores y Jóvenes en Riesgo de Abuso y Dependencia de Alcohol y otras Drogas. 2014. Disponible en: https://riuma.uma.es/xmlui/handle/10630/7432

7. Urra, E., Retamal, C., Tapia, C. y Rodríguez, M. Enfermería basada en la evidencia: qué es, sus características y dilemas. Investigación y Educación en Enfermería. 2010; 8 (1): 108-118: Disponible en: http://www.redalyc.org/articulo.oa?id=105215294014

8. Madruga CS, Laranjeira R, Caetano R, Pinsky I, Zaleski M, Ferri CP. Use of licit and illicit substances among adolescents in Brazil--A national survey. Addict Behav. 2012; 37(10):1171-5. doi: 10.1016/j.addbeh.2012.05.008

9. Barrios-Ayola F., Hurtado-Acosta A. Prevalencia del consumo compulsivo de alcohol y sustancias ilícitas en pacientes hospitalizados por patologías psiquiátricas. Revista Ciencias Biomédicas. 2012; 3(1). Disponible en: http://revistas.unicartagena.edu.co/index.php/cienciasbiomedicas/article/view/1081

10. Lev Ran, S., et al. Clinical correlates of cannabis use among adolescent psychiatric inpatients. European Psychiatry. 2012; 27( 6): 470-475. Disponible en: http://www.scielo.cl/pdf/rchnp/v51n4/art05.pdf

11. Galera, C. et al. Síntomas psiquiátricos asociados al consumo de pasta base de cocaína en niños y adolescentes: un estudio exploratorio en Montevideo, Uruguay. Rev. chil. neuro-psiquiatr. 2013; 51 (4). Disponible en: http://www.scielo.cl/pdf/rchnp/v51n4/art05.pdf

12. Esfors J. et al. Unidad Hospitalaria de Patología Dual Grave: un año de experiencia. Revista Española de Drogodependencias. 2014; 39(1). Disponible en: http://roderic.uv.es/handle/10550/47630

13. Gálvez JF, Rincón DA.Manejo clínico de pacientes con diagnóstico dual. Evaluación diagnóstica de los pacientes farmacodependientes que sufren de comorbilidad psiquiátrica. Revista Colombiana de Psiquiatría. 2008; 37(3): 397-417. Disponible en: http://www.redalyc.org/articulo.oa?id=80611205010

14. Arévalo MT, Torres CI, Rodríguez ED, Cuevas RJ. Consumo de sustancias psicoactivas ilegales en jóvenes: factores psicosociales asociados. Pensamiento Psicológico. 2007; 3(8):31-45. Disponible en: http://revistas.javerianacali.edu.co/index.php/pensamientopsicologico/article/view/70/208

15. Moral MV, Rodríguez FJ, Sirvent C. Factores relacionados con las actitudes juveniles hacia el consumo de alcohol y otras sustancias psicoactivas. Psicothema. 2006; 18(1):52-58. Disponible en: http://www.psicothema.es/pdf/3175.pdf

16. Gómez-Restrepo C. et al. Prevalencia del consumo de sustancias psicoactivas en pacientes no Psiquiátricos que consultan un servicio de urgencias en Bogotá. Revista Colombiana de Psiquiatría. 2011; 40(4): 709-722. Disponible en: https://www.sciencedirect.com/science/article/pii/S0034745014601591 


\section{Revista Electrónica Enfermeria Actual en costa Rica}

17. Arias F., et al. Abuso o dependencia al cannabis y otros trastornos psiquiátricos. Estudio Madrid sobre prevalencia de patología dual. Actas Esp Psiquiatr 2013;41(2):122-9. Disponible en: https://www.actaspsiquiatria.es/repositorio/15/82/ESP/15-82-ESP-122-129-974945.pdf

18. Madoz-Gúrpide A., García V., Luque E., Ochoa E. Variables predictivas de alta terapéutica entre pacientes con patología dual grave atendidos en una comunidad terapéutica de drogodependencias con unidad psiquiátrica. $\quad$ Adicciones. 2013; 25(4). Disponible http://www.adicciones.es/index.php/adicciones/article/view/30

19. Casado V., Araúzo D. Intervención en drogas desde atención primaria: revisión de guías clínicas. Adicciones. 2004; 16(3). Disponible en: http://www.adicciones.es/index.php/adicciones/article/view/404

20. Del Pozo Para, Esther. Programa de promoción de la Salud Mental dirigido a la población adolescente en la escuela. Biblioteca Las casas, 2006; 2(4) Disponible en: http://www.indexf.com/lascasas/documentos/lc0180.pdf

21. González A. Prevalencia y elementos sociodemográficos asociados a los trastornos mentales más frecuentemente diagnosticados a los adolescentes internados por primera vez en el pabellón de adolescentes del hospital nacional psiquiátrico durante el primer semestre del 2015 [Tesis]. Costa Rica: Universidad de Costa Rica; 2015. Disponible en: http://www.binasss.sa.cr/bibliotecas/bhp/textos/tesis70.pdf

22. World Health Organization. Mental health: strengthening mental health promotion. Fact Sheet 220, revised, November 2016. Disponible en: www.who.int/mediacentre/factsheets/ fs220/en/print.html.

Contribución de la autoría de este trabajo

\begin{tabular}{|l|l|}
\hline Resumen del protocolo & Carlos E. Marín Madrigal \\
\hline Desarrollo de las estrategias de búsqueda & Jose M. Calderón Barboza \\
\hline Selección de estudios incluidos & Carlos E. Marín Madrigal \\
\hline
\end{tabular}




\begin{tabular}{|l|l|}
\hline Extracción de los datos de los estudios & Carlos E. Marín Madrigal y Jose M. Calderón Barboza \\
\hline Elaboración del análisis & Jose M. Calderón Barboza \\
\hline
\end{tabular}

